\title{
A Rhodamine Chemodosimeter Bearing Thiourea Moiety for Mercury(II) and Its Bioimaging Application
}

\author{
A. Tantipanjaporn ${ }^{123}$, P. Kongsaere ${ }^{123}$ \\ ${ }^{1}$ Department of Chemistry, Faculty of Science, Mahidol University, Bangkok, Thailand, \\ ${ }^{2}$ Center for Excellence in Protein and Enzyme Technology, Faculty of Science, Mahidol University, \\ Bangkok, Thailand, \\ ${ }^{3}$ Center of Excellence for Innovation in Chemistry, Faculty of Science, Mahidol University, Bangkok, \\ Thailand \\ palangpon.kon@mahidol.ac.th
}

\begin{abstract}
:
A selective rhodamine chemodosimeter RTP was developed for $\mathrm{Hg}^{2+}$ detection based on the $\mathrm{Hg}^{2+}$-promoted intramolecular cyclic guanylation of thiourea linked with o-phenylenediamine as a linker. Upon the addition of $\mathrm{Hg}^{2+}$, a colorless solution of RTP turned pink with a maximum absorption band at $555 \mathrm{~nm}$ and with a 62-fold fluorescence enhancement at $578 \mathrm{~nm}(\Phi=0.34)$. RTP is significantly selective to $\mathrm{Hg}^{2+}$ among other metal ions with a detection limit of $1.6 \mathrm{nM}(0.3 \mathrm{ppb})$. The membrane-permeable RTP probe was successfully demonstrated in $\mathrm{Hg}^{2+}$ monitoring in cultured HeLa cells.
\end{abstract}

Key words: bioimaging, fluorescence detection, $\mathrm{Hg}^{2+}$-selective chemodosimeter, rhodamine, thiourea

\section{Introduction}

A number of $\mathrm{Hg}^{2+}$-selective chemosensors have been reported allowing the sensitive detection of mercury ions [1]. However, various downsides that may limit the practical use of chemosensors are low aqueous solubility, low quantum yield in aqueous media, and cross-sensitivity to other metal cations. Thus, the development of novel $\mathrm{Hg}^{2+}$-selective chemosensors is still a challenge. Herein, we report the thiourea-appended fluorescent chemodosimeter RTP for $\mathrm{Hg}^{2+}$ detection with o-phenylenediamine as a linker. Binding between $\mathrm{Hg}^{2+}$ and thiourea would promote desulfurization of thiourea leading to the spirolactam ring opening and intrinsic fluorescence emission.

\section{Synthesis}

The production of rhodamine-phenylthiourea conjugate RTP was synthesized in 2 steps with $51 \%$ yield (Fig. 1).

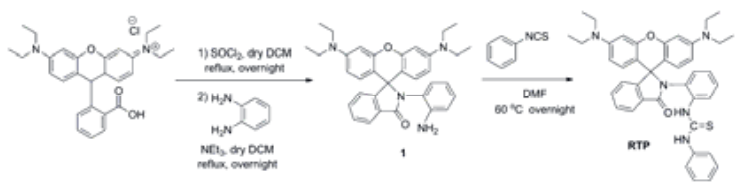

Fig. 1. Synthesis of RTP.

The structures of RTP and 1 were spectroscopically established by ${ }^{1} \mathrm{H}$ NMR, ${ }^{13} \mathrm{C}$ NMR, FT-IR and HRESI-MS analysis. In addition, the structure of RTP was also confirmed by a single-crystal X-ray crystallographic analysis showing spirolactam ring is in the closed form (Fig. 2).

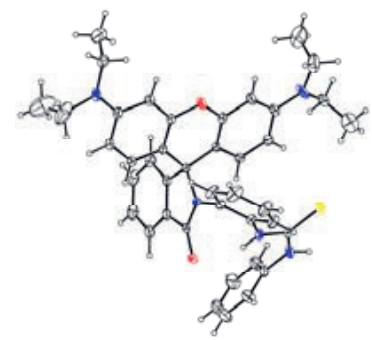

Fig. 2. ORTEP plot of the chemodosimeter RTP.

\section{Optical and Fluorescence Responses}

A colorless solution of RTP responded to $\mathrm{Hg}^{2+}$ by turning pink with a strong fluorescence emission $(\Phi=$ 0.37 , using rhodamine $B$ as a standard in $1: 9$ $\mathrm{CH}_{3} \mathrm{CN}$-HEPES) which the optical change can be visualized by naked eyes. Moreover, solutions of RTP $(10 \mu \mathrm{M})$ was also examined with various metal ions $(10 \mu \mathrm{M})$ in $\mathrm{CH}_{3} \mathrm{CN}$-HEPES buffer $(1: 9, \mathrm{v} / \mathrm{v}, 50$ $\mathrm{mM} \mathrm{pH} \mathrm{7.2)} \mathrm{including} \mathrm{competitive} \mathrm{experiment} \mathrm{using}$ fluorometric technique. After $\mathrm{Hg}^{2+}$ addition, the fluorescent intensity was clearly visible while other metals did not cause any significant change in the intensity (Blue bar, Fig. 3). The results suggested that the spirolactam ring was only opened upon binding of $\mathrm{Hg}^{2+}$. In the presence of miscellaneous competitive cations, $\mathrm{Hg}^{2+}$ still caused the higher fluorescence change (Red bar, Fig. 3). Obviously, the increase of the fluorescence intensity by the 
addition of $\mathrm{Hg}^{2+}$ was not influenced by the subsequent addition of those cations.

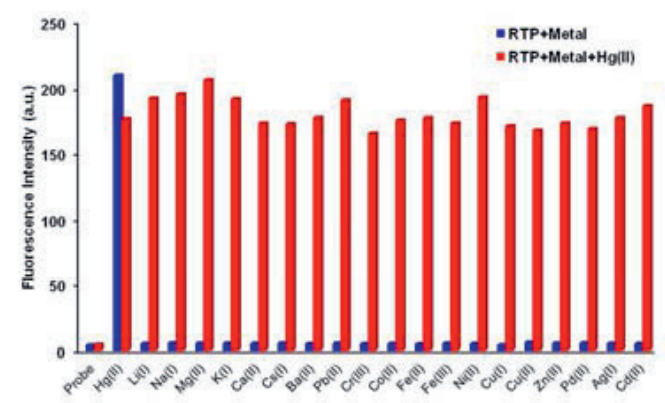

Fig. 3. Fluorescence response of RTP $(10 \mu \mathrm{M})$ in the selectivity and the competitive experiments with various metal ions $\left(\lambda_{e x}=550 \mathrm{~nm}\right.$. $\left.\lambda_{e m}=578 \mathrm{~nm}\right)$.

\section{Fluorescent titration}

Fluorescence titration of RTP $(10 \mu \mathrm{M})$ with $\mathrm{Hg}^{2+}(0-$ $30 \mu \mathrm{M})$ revealed that fluorescence intensity of RTP was linearly proportional to the concentration of $\mathrm{Hg}^{2+}$ in the range of 0-8 $\mu \mathrm{M}$ (Fig. 4). The limit of detection (LOD) for $\mathrm{Hg}^{2+}$ of RTP is $1.6 \mathrm{nM}(0.3 \mathrm{ppb})$.

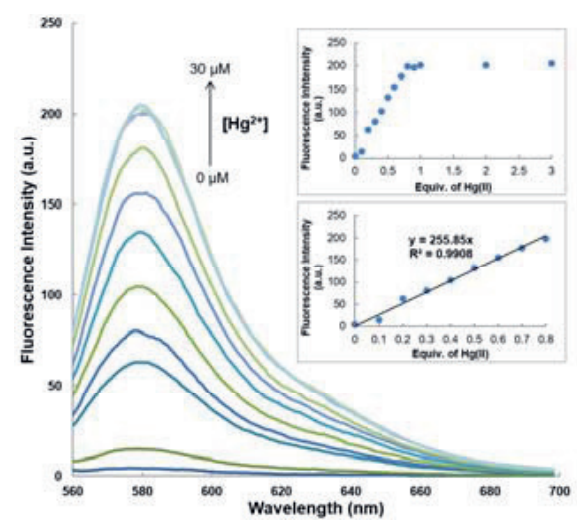

Fig. 4. Fluorescence titration spectra of RTP $(10 \mu \mathrm{M})$ upon the addition of increasing $\mathrm{Hg}^{2+}$ concentrations (0-30 $\mu \mathrm{M})$ in $\mathrm{CH}_{3} \mathrm{CN}$-HEPES buffer (1:9, v/v, pH 7.2) by excitation at $550 \mathrm{~nm}$.

\section{Sensing mechanism}

An ESI-MS analysis of the mixing of RTP and excess $\mathrm{Hg}^{2+}$ disclosed three distinct molecular ions at $\mathrm{m} / \mathrm{z}$ $634.3239,457.2557$ and 210.1029. Upon binding of $\mathrm{Hg}^{2+}$ with the thiourea sulfur atom, the $\mathrm{N}$ atom of the spirolactam was induced to react with the thiourea carbon and hence caused the ring opening of the spirolactam. After HgS removal and intramolecular guanylation, the benzimidazole appended rhodamine intermediate 2 (calcd for $\mathrm{C}_{41} \mathrm{H}_{40} \mathrm{~N}_{5} \mathrm{O}_{2} \quad \mathrm{~m} / \mathrm{z}=$ 634.3182) was observed [2]. The benzimidazole moiety, as a good leaving group, underwent a hydrolysis reaction to yield the corresponding rhodamine methyl ester 3 (calcd for $\mathrm{C}_{29} \mathrm{H}_{33} \mathrm{~N}_{2} \mathrm{O}_{3} \mathrm{~m} / \mathrm{z}$ = 457.2491) and 2-(4-aminophenyl) benzimidazole 4 (calcd for $\mathrm{C}_{13} \mathrm{H}_{12} \mathrm{~N}_{3} \mathrm{~m} / \mathrm{z}=210.1031$ ), respectively (Fig. 5).

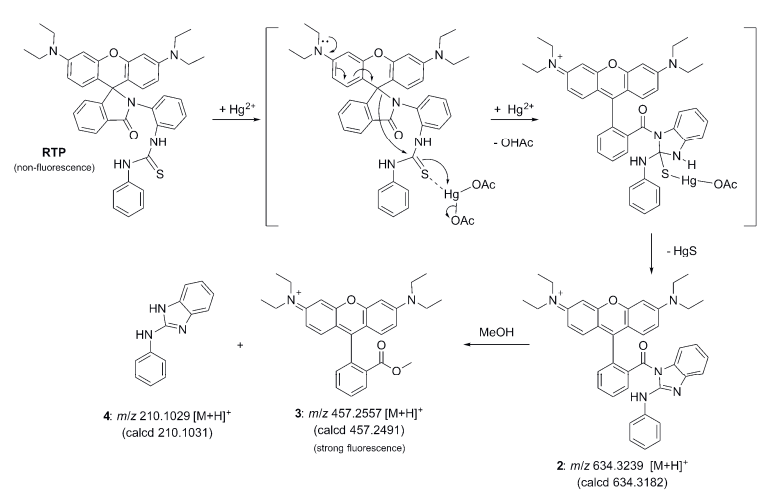

Fig. 5. Proposed mechanism of $\mathrm{Hg}^{2+}$-promoted ring opening of RTP.

\section{Fluorescent imaging}

Bioimaging of HeLa cells incubated with only RTP probe revealed the cell morphology was not harmed in this event (a-e, Fig. 6). Evidently, the cells incubated with RTP and then $\mathrm{Hg}^{2+}$ exhibited an intense fluorescence emission (e-h, Fig. 6). The result clearly demonstrated that the RTP probe could permeate the cell membrane and showed a specific fluorescence emission only in presence of $\mathrm{Hg}^{2+}$.

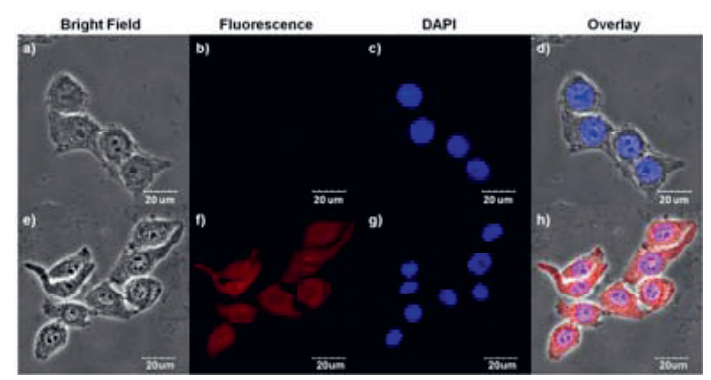

Fig. 6. Confocal fluorescence images. The HeLa cells were incubated with $\mathrm{Hg}^{2+}(30 \mu \mathrm{M})$ in DPBS buffer, $1 \mathrm{~h}(\mathrm{e}-\mathrm{h})$ and then incubated with RTP (10 $\mu M), 1 h$ (a-h). (a, e) Bright-field images; $(b, f)$ fluorescence images; $(c, g)$ fluorescence images stained with DAPI; $(d, h)$ merged images.

\section{Conclusion}

RTP was a highly selective fluorogenic chemodosimeter to $\mathrm{Hg}^{2+}$ with a detection limit of 1.6 $\mathrm{nM}(0.3 \mathrm{ppb}) . \mathrm{Hg}^{2+}$ promoted desulfurization of the probe and caused the ring-opening of the spirolactam in an irreversible fashion. Furthermore, RTP could be a beneficial fluorescence probe for bioimaging of $\mathrm{Hg}^{2+}$.

\section{References}

[1] P. Mahato, S. Saha, P. Das, H. Agarwalla and A. Das, An Overview of the Recent Developments on $\mathrm{Hg}^{2+}$ Recognition, RSC Advances 4, 3614036174 (2014); doi: 10.1039/C4RA03594A

[2] X. Chen, T. Pradhan, F. Wang, J. S. Kim and J. Yoon, Fluorescent Chemosensors Based on Spiroring-Opening of Xanthenes and Related Derivatives, Chemical Reviews 112, 1910-1956 (2012); doi: 10.1021/cr200201z 